\title{
Bases Ideológicas de la Percepción del Sistema Normativo y el Cinismo Político en Adultos de Sectores Urbanos del Perú
}

\section{Ideological Bases of Perception of the Normative System and Political Cynicism in Adults From Urban Sectors of Peru}

\author{
Erika Janos, Agustín Espinosa y Manuel Pacheco \\ Pontificia Universidad Católica del Perú
}

\begin{abstract}
El propósito fue analizar la influencia de expresiones ideológicas conservadoras, como el autoritarismo de derecha (RWA) y la orientación hacia la dominancia social (SDO), en las percepciones sobre el sistema normativo y el sistema político en el Perú en una muestra accidental de 201 peruanos. Se aplicaron las escalas RWA, SDO, percepción del sistema normativo y cinismo político. Por medio de análisis de varianza, $t$ de Student, análisis factorial exploratorio, correlaciones de Pearson, regresión lineal múltiple y path analysis, los resultados muestran, a nivel descriptivo, que las percepciones sobre los sistemas normativo y político son mayoritariamente negativas. En general, se observan relaciones directas entre los indicadores de autoritarismo y dominancia, con una evaluación positiva del sistema normativo y con una mayor tolerancia hacia prácticas corruptas en el sistema político y sus instituciones. Se parte de una definición del cinismo político como una expresión de desconfianza generalizada hacia el sistema político, que se enriquece con 2 nuevas dimensiones conceptuales relevantes para la comprensión del fenómeno en sociedades latinoamericanas como la peruana: posibilidad de cambio del sistema político y laxitud moral política.
\end{abstract}

Palabras clave: percepción sistema normativo, dominancia social, autoritarismo de derecha, cinismo político, path analysis

\begin{abstract}
The purpose of this study was to analyze the influence of conservative ideological expressions like right wing authoritarianism (RWA) and social domination orientation (SDO) on perceptions related to the normative and political system in Peru in an accidental sample of 201 Peruvians. The RWA, SDO, normative system perception, and political cynicism scales were administered. Based on variance analyses, Student's $t$ tests, exploratory factor analyses, Pearson correlations, multiple linear regressions, and path analysis, descriptive results show that perceptions on the normative and political systems are mostly negative. In general, results reveal direct relationships between the authoritarianism and dominance indicators and a positive evaluation of the normative system and greater tolerance of corrupt practices in the political system and its institutions. The study rests on a definition of political cynicism as an expression of generalized mistrust towards the political system, which is enriched with 2 new conceptual dimensions relevant for understanding the phenomenon in Latin American societies such as the Peruvian: the possibility of change in the political system and political moral laxity.
\end{abstract}

Keywords: normative system perception, social domination, right-wing authoritarianism, political cynicism, path analysis

En el contexto peruano existe una larga historia de corrupción y transgresión normativa (Quiroz, 2008/2013), produciéndose una suerte de precarización del sistema político y las normas que lo respaldan, lo que, a su vez, se traduce en muy bajos niveles de confianza institucional (Latinobarómetro, 2015). Al ser compartida por gran parte de la sociedad, esta percepción negativa del sistema normativo y político da lugar a una desconfianza generalizada que puede ser entendida como cinismo político, el cual tiene consecuencias que terminan por amenazar la democracia. Asimismo, la literatura sugiere que la ideología conservadora, en sus dimensiones autoritaria y dominante, tiende a agravar la situación de cinismo político y percepción negativa del sistema normativo (Schmitz \& Espinosa, 2015), por lo que el estudio de las relaciones entre estas variables resulta pertinente.

Erika Janos, Agustín Espinosa y Manuel Pacheco, Grupo de Psicología Política y Social, Departamento de Psicología, Pontificia Universidad Católica del Perú, Lima, Perú.

La correspondencia relativa a este artículo debe ser dirigida a Erika Janos, Departamento de Psicología, Pontificia Universidad Católica del Perú, Avenida Universitaria 1801, San Miguel, Lima 15088, Perú. E-mail: ejanos@pucp.pe 


\section{Percepción del Sistema Normativo e Ideología Política}

Las normas, configuradas por costumbres y valores, describen y orientan el comportamiento humano, brindando estándares de conducta que facilitan la convivencia en sociedad. Sin embargo, para que se pueda dar una imposición de patrones de comportamiento aceptados socialmente es necesario un sistema de poder confiable que regule el comportamiento de los individuos (Beramendi \& Zubieta, 2013). Así, el cumplimiento voluntario de las normas en una sociedad dependerá, entonces, de la percepción del sistema normativo vigente en la misma, el cual debería ser considerado como legítimo y justo para garantizar su adecuado funcionamiento (Beramendi \& Zubieta, 2014; Rottenbacher \& Schmitz, 2012).

De acuerdo a Tyler (2001), la legitimidad percibida en el sistema normativo incluye una valoración del rol de las autoridades e instituciones que establecen las normas y su cumplimiento dentro de una sociedad. Se constituye sobre la base de un conjunto de percepciones acerca del funcionamiento del sistema político y su capacidad de administrar justicia y distribuir riqueza de forma eficaz y eficiente (Kelman, 2001; Major \& Schmader, 2001; Tyler, 2006; Zelditch Jr., 2001).

En este escenario, la ideología política aparece como un constructo relevante para comprender las percepciones de las personas sobre el sistema normativo, pues la ideología parece tener efecto en la forma en que los individuos interpretan las normas, determinando las formas y niveles en que se justifican acciones de transgresión o se promueven acciones de respeto normativo en una sociedad (Gnädinger, 2014).

Desde una aproximación psicopolítica, la ideología es entendida como un conjunto de actitudes, creencias y valores con propiedades cognitivas, afectivas y motivacionales (Jost, 2006; Knight, 2006). La ideología es socialmente compartida y provee a las personas de un marco de interpretación del mundo social, influyendo en los contenidos de sus propias creencias y en la orientación de sus actitudes sociales y políticas (Brown, 2004).

Desde una perspectiva multidimensional, el estudio de la ideología política establece que el conservadurismo político en las personas puede entenderse a través de un enfoque dual (Altemeyer, 1998; Duckitt \& Fisher, 2003; Duckitt \& Sibley, 2007; Duckitt, Wagner, du Plessis \& Birum, 2002; Jost, Federico \& Napier, 2009; Pratto, Sidanius, Stallworth \& Malle, 1994; Van Hiel \& Mervielde, 2002), comprendido por los constructos de autoritarismo de derecha (RWA por su sigla en inglés) y de orientación hacia la dominancia social (SDO por su sigla en inglés), los cuales sugieren que el conservadurismo político se expresa en dos vertientes moderadamente relacionadas, pero motivacionalmente diferentes entre sí, y que se contraponen a una única dimensión ideológica expresada en valores liberales e igualitarios (Altemeyer, 1998).

De manera específica, el RWA está compuesto por tres elementos actitudinales: la sumisión a la autoridad y a las normas sociales que estas autoridades representan, una predisposición a la agresión autoritaria y un alto convencionalismo (Altemeyer, 1998; Duriez, Van Hiel \& Kossowska 2005; Stone, 2001).

Empíricamente, se ha establecido que existe una tendencia de aquellas personas más conservadoras autoritarias a percibir el mundo como un lugar amenazante y peligroso, lo cual las impulsa a buscar medidas de control social, aunque ello implique el uso de la fuerza y de la violencia (Duckitt 2001; Jackson \& Gaertner, 2010; Jost, Glaser, Kruglanski \& Sulloway, 2003; Jost et al., 2009). Esto volvería a las personas autoritarias más susceptibles a respetar las normas que impone el entorno, siempre y cuando las normas sean coherentes con su visión conservadora y deriven de autoridades e instituciones percibidas como legítimas. Sin embargo, si las normas atentan contra su visión social del mundo o provienen de instancias no legitimadas por esta visión, tornándose una amenaza, se adhieren a una posición contraria a las mismas. Entonces, la amenaza percibida vuelve a los individuos autoritarios más propensos a una desconexión moral, justificando sus acciones violentas o transgresoras (Jackson \& Gaertner, 2010).

Por otro lado, quienes presentan altas puntuaciones en la SDO tenderán a aprobar la promoción y mantenimiento de políticas y acciones que refuercen las jerarquías y asimetrías de poder entre grupos e individuos, así como tenderán a una preferencia por la superioridad y dominio de grupos de alto estatus ante grupos de bajo estatus, independientemente de su afiliación grupal (Altemeyer, 1998; Sidanius, Pratto \& Bobo, 1996).

Niveles altos de SDO dan lugar a deseos de poder y superioridad que reducen la preocupación por los demás, mostrando mayor hostilidad y tendencia a la transgresión de las normas, especialmente cuando son consideradas impedimentos para lograr la consolidación de las propias capacidades de dominio (Rottenbacher \& Schmitz, 2012). Empíricamente, se han encontrado correlaciones inversas entre la dominancia y la empatía, la tolerancia y el altruismo (Pratto et al., 1994), por lo que aquellos individuos más dominantes son 
más propensos a la difusión de la responsabilidad ante decisiones violentas tomadas por una autoridad y, también, tienden a una desvinculación moral ante la transgresión de ciertas normas sociales y la aceptación de la violencia (Jackson \& Gaertner, 2010).

\section{Cinismo Político, Percepción del Sistema Normativo e Ideología Política}

Si existe desconfianza en los gobernantes y en su gestión del sistema normativo, compartida por un gran número de personas y mantenida por un largo tiempo, esto puede dar lugar a la prevalencia de un fenómeno denominado cinismo político (Beramendi \& Zubieta, 2014). De manera general, el cinismo político revela un descrédito hacia el sistema político. La literatura sugiere tres elementos o dimensiones que motivan el cinismo político: (a) un sentimiento de impotencia en las personas para influir en la política; (b) una desconfianza hacia la política en general, las instituciones y los actores políticos que lo conforman y (c) una percepción generalizada de que el sistema político y las normas que lo regulan son débiles, injustas o ineficientes. En este sentido, la corrupción y la falta de compromiso con la representación de los intereses de las personas incrementarían el descrédito de los políticos (Miller,1974; Siu-kai, 1992).

Lo anterior termina por traducirse en una desconfianza generalizada, que influye en una mayor tendencia a percibir el sistema normativo como carente de legitimidad (Beramendi \& Zubieta, 2014; Strama, 1998). Además, el cinismo como actitud política conlleva, paradójicamente, a una resistencia al cambio en el sistema y a un enfoque negativo y escéptico sobre la política (Fu, Mou, Miller \& Jalette, 2011).

Adicionalmente, las consecuencias del cinismo político son consideradas potencialmente peligrosas para la democracia, pues tiene el poder de reducir la participación ciudadana en el ámbito político (Patterson, 2002) e incrementar el soporte a medidas extremas de oposición (Bélanger \& Aarts, 2006). Como contraparte, el clima de cinismo fuerza a los líderes políticos a tomar decisiones más débiles y evitar problemas sociales urgentes pero controversiales, haciendo más difícil la solución de las problemáticas nacionales (Porter, 2007).

De manera complementaria al entendimiento clásico del cinismo político, estudios en Latinoamérica han logrado identificar una tendencia a la aceptación de prácticas corruptas, incorporando cierta laxitud moral como elemento de la identidad, lo cual podría resultar uno de los elementos más peligrosos del cinismo político. Este fenómeno parece presentarse de manera más importante en aquellos ciudadanos más dominantes (Monsegur, Espinosa \& Beramendi, 2014). De esta forma, la literatura sugiere que ciertas manifestaciones de ideología conservadora, en sus dimensiones autoritaria y dominante, tienden a agravar la situación de cinismo político y percepción negativa del sistema normativo, lo que supondría la consolidación de un círculo vicioso (Schmitz \& Espinosa, 2015).

En el contexto peruano la historia de corrupción y transgresión normativa son de larga data. Así, el abuso de los recursos públicos en beneficio de unos cuantos se ha dado a costa del progreso general, público e institucional de la nación, deteriorando en la opinión pública la percepción de las instituciones públicas y políticas y consolidando una "naturalización" de las prácticas corruptas como "inevitables" en el sistema (cf. Quiroz, 2008/2013). Consistentemente con lo anterior, la precarización del sistema político y las normas que lo respaldan se traducen en muy bajos niveles de confianza institucional hacia el poder judicial $-76,9 \%$ tiene poca o ninguna confianza en la instancia que administra la justicia en el país- y hacia el poder ejecutivo - 74,6\% tiene poca o ninguna confianza en la instancia que ejecuta las normas en el país(Latinobarómetro, 2015).

Estos resultados han sido corroborados en un estudio transcultural que muestra que en aquellos sistemas donde predomina la percepción negativa del sistema y sus instituciones, en términos de corrupción y transgresión, se afectan en mayor medida aspectos psicológicos que comprenden la honestidad intrínseca de las personas en una sociedad, tornándolas más propensas a aceptar prácticas que afectan la convivencia y el contrato social por la obtención de beneficios individuales (cf. Gächter \& Schulz, 2016). Adicionalmente, Gnädinger (2014) y Monsegur et al. (2014) encuentran que hay aspectos ideológicos, normalmente relacionados con la dominancia social, que se asocian directamente con la tendencia a considerar como legítimas prácticas que van desde pequeñas transgresiones hasta actos de corrupción.

En este escenario, el objetivo general de la presente investigación fue describir y analizar las relaciones existentes entre las dimensiones correspondientes a los indicadores sobre ideología política en sus fases autoritaria y dominante, la percepción del sistema normativo y la expresión de cinismo político en una muestra de ciudadanos peruanos de Lima Metropolitana y otras ciudades del país. De manera complementaria, se analizó la estructura factorial de la escala de cinismo político desarollada para el estudio. 
JANOS, ESPINOSA Y PACHECO

\section{Método}

\section{Participantes}

La muestra estuvo compuesta por 201 peruanos de las ciudades de Amazonas, Ancash, Apurímac, Arequipa, Cajamarca, Cuzco, Huánuco, Ica, Junín, La Libertad, Lambayeque, Loreto, Pasco, Piura y San Martín, de entre 18 y 89 años $(M=30,90, D E=13)$, siendo 101 varones $(50,2 \%)$ y 100 mujeres $(49,8 \%)$. En relación al nivel educativo, 113 de los participantes poseían estudios universitarios de pregrado (56,2\%), 58 tenían estudios universitarios de posgrado (28,9\%), y el resto estaba dividido entre aquellos que solo tenían secundaria $(9 \%)$ o estudios superiores técnicos (6\%). En cuanto al nivel socioeconómico autopercibido, la mayoría se ubica en un sector de clase media típica (52,7\%), seguido por el sector de clase media alta $(26,4 \%)$ y media baja $(13,9 \%)$ y, finalmente, los extremos de clase alta $(6,5 \%)$ y baja $(0,5 \%)$.

Para la selección de los participantes se utilizó un muestreo accidental, haciendo una convocatoria abierta a través de redes sociales (Facebook y correo electrónico).

En cuanto a las variables de inclusión en el estudio, se consideraron específicamente tres: (a) los participantes tenían que tener nacionalidad peruana, (b) debían residir actualmente en el país y (c) debían tener más de 18 años (ser mayores de edad).

\section{Instrumentos}

Medidas sociodemográficas. Se utilizó una ficha demográfica que recolectaba datos en relación a la edad, sexo, nivel educativo, nivel socioeconómico autopercibido, nacionalidad y país de residencia.

Escala de Percepción del Sistema Normativo. Se utilizó la versión elaborada en Argentina por Beramendi y Zubieta (2014), la cual cuenta con 20 ítems que son respondidos en una escala Likert del 1 al 7, en donde 1 es Totalmente en desacuerdo y 7 es Totalmente de acuerdo. Una puntuación elevada refiere a una percepción negativa del sistema normativo. Se realizó un análisis de validez convergente utilizando otras escalas que se relacionan teóricamente con la misma, obteniendo resultados positivos y congruentes (Beramendi \& Zubieta, 2014). La versión utilizada en el presente estudio es la adaptada por Gnädinger (2014) para una investigación con adultos de sectores urbanos de nivel socioeconómico medio en el Perú. La escala cuenta con tres dimensiones: (a) Falta de Legitimidad, relacionada con la percepción de ausencia de legitimidad, tanto de autoridades como de instituciones, con una consistencia interna, evaluada con alfa de Cronbach, de a = 0,67 y que comprende 10 ítems, tales como "En este país es raro que se lleven a cabo juicios justos"; (b) Transgresión, que tiene que ver con la percepción de la presencia de conductas transgresoras en la sociedad por parte de los ciudadanos, y que se expresa en seis ítems, tales como "Los peruanos siempre encuentran la manera de transgredir las normas", con una consistencia interna de $\alpha=0,75$ y (c) Debilidad Normativa, que se asocia a una evaluación negativa de cómo están conformadas y construidas las normas, percibidas estas como débiles e ineficientes, y que se expresan en cuatro ítems del tipo "En las instituciones siempre hay una vía formal que se debería seguir y una vía irregular que agiliza los procesos", con una consistencia interna de $\alpha=0,55$.

Escala Resumida de Autoritarismo de Derecha (RWA). Se utilizó, de manera unidimensional, una versión traducida al castellano y adaptada por Rottenbacher y Schmitz (2012) de la versión de la Escala Resumida de Autoritarismo de Derecha de Zakrisson (2005). Esta versión ha sido utilizada en estudios previos con muestras urbanas de sectores socioeconómicos medios en Lima, obteniéndose resultados positivos sobre la validez convergente de la misma (e.g., Rottenbacher \& Schmitz, 2012). Este instrumento está compuesto por 15 ítems, como, por ejemplo: "Nuestro país necesita un líder poderoso para erradicar las tendencias radicales e inmorales prevalentes en la sociedad actual". La opción de respuesta a cada ítem se presenta en una escala Likert de 7 puntos que van de $1=$ Totalmente en desacuerdo a $7=$ Totalmente de acuerdo. Las puntuaciones más elevadas en la escala sugieren mayores niveles de autoritarismo de derecha en los participantes. En este estudio se obtuvo un alfa de Cronbach de 0,69.

Escala de Orientación hacia la Dominancia Social (SDO). Se utilizó, de manera unidimesional, la versión traducida al castellano por Montes-Berges y Silván-Ferrero (2003, citado en Moya \& MoralesMarente, 2005) de la Escala de Orientación hacia la Dominancia Social (Sidanius \& Pratto, 1999, citado en Sidanius, Pratto, Van Laar \& Levin, 2004). Esta versión ha sido utilizada en diversos estudios con muestras 
de sectores urbanos del Perú, obteniendo evidencia positiva sobre la validez convergente de la escala (e.g., Espinosa, 2011). Esta escala consta de 16 enunciados referidos a relaciones jerárquicas y asimétricas entre grupos pertenecientes a una misma sociedad, como, por ejemplo: "La diferencia entre grupos sociales es natural y debería ser mantenida". La opción de respuesta es una escala Likert de 7 puntos que van desde $1=$ Totalmente en desacuerdo a $7=$ Totalmente de acuerdo. Una mayor puntuación en la escala refiere a mayores niveles de acuerdo con la ideología conservadora dominante. En este estudio se obtuvo un alfa de Cronbach de 0,90 .

Escala de Cinismo Político. Se aplicó una escala desarrollada para el presente estudio. Se construyó un banco de ítems con base en información disponible sobre la situación política del país. Luego se aplicaron diversos estudios piloto donde se identificaron los ítems más claros en su contenido. La escala quedó compuesta por 16 ítems (ver estos en la Tabla 2), respondidos en una escala Likert de 1 a 4, en donde 1 es Totalmente en desacuerdo y 4 es Totalmente de acuerdo. Para la versión actual se presenta un análisis de la estructura factorial para detectar si la agrupación de los ítems corresponde a los elementos de la teoría sobre el tema. Dicha información será reportada en el acápite de Resultados.

\section{Procedimiento}

Los cuestionarios aplicados fueron digitalizados mediante la herramienta de Google Drive, generando, así, una encuesta online. Se decidió hacerlo de esta forma ya que se buscaba que la mayor cantidad de personas contestaran los instrumentos, sin importar el lugar del país en el que se encontraban. A los participantes se les presentaba de forma inicial en su pantalla un consentimiento informado, en el que se les indicaba la naturaleza del estudio, información de los aspectos éticos, como el anonimato y la confidencialidad, así como de la libertad de dejar de responder el cuestionario si en algún momento así se deseaba. Una vez que los participantes culminaban de leer el consentimiento informado, manifestaban su acuerdo a responder la encuesta dando click al comando correspondiente para el inicio de la misma. Cuando terminaban, enviaban sus respuestas, generándose una hoja Excel que luego fue importada al software SPSS para su posterior análisis.

\section{Análisis de Datos}

Inicialmente, se revisó la consistencia interna de las dimensiones de todas las escalas, utilizando el coeficiente alfa de Cronbach. Seguidamente, se efectuó un análisis descriptivo de las dimensiones de la Escala de Percepción del Sistema Normativo y de las escalas de ideología política. Específicamente, para ver si existieron diferencias entre las dimensiones intra-escala, se realizaron comparaciones de medias, por medio de un análisis de varianza de medidas repetidas y utilizando la prueba post hoc de Bonferroni ajustado para las comparaciones múltiples, así como una prueba $t$ de Student para muestras dependientes. En ambos casos se midió el tamaño del efecto, usando eta cuadrada parcial para el primer procedimiento y $d$ de Cohen para el segundo. Para indagar en la estructura de la Escala de Cinismo Político se efectuó un análisis factorial exploratorio. El método de extracción utilizado fue el de componentes principales y el método de rotación fue Varimax con normalización de Kaiser. Luego, teniendo los factores identificados, se realizó un análisis descriptivo de cada una de las dimensiones.

Para lograr el objetivo principal, se realizaron correlaciones lineales de Pearson entre todas las dimensiones de la ideología política, Percepción del Sistema Normativo y Escala de Cinismo Político. Para interpretar las magnitudes del efecto, se utilizó el criterio de Cohen, que indica que hay tres intensidades de asociación: pequeña $(0,1 \leq|r| \leq 0,3)$, mediana $(0,3 \leq|r| \leq 0,5)$ y grande $(|r|>0,5)$ (Cohen, 1988). Además, se plantearon diversos modelos de regresión con el fin de explicar la influencia de la ideología política sobre la Percepción del Sistema Normativo, así como del Cinismo Político.

Se elaboraron ocho modelos de regresión lineal múltiple, utilizando el método "por pasos", considerando como variables independientes a la SDO y el RWA y como variables dependientes, las tres dimensiones de la Percepción del Sistema Normativo y las cinco dimensiones de Cinismo Político. Así, también, con la intención de conocer los mejores predictores de la Percepción del Sistema Normativo del Cinismo Político, se realizaron adicionalmente cinco modelos de regresión con el método "por pasos". En este segundo conjunto de análisis, se consideraron como variables independientes las tres dimensiones de la Percepción del Sistema Normativo y como variables dependientes, cada uno de los cinco factores del Cinismo Político. 
Para analizar de manera global las múltiples relaciones entre las variables estudiadas se realizó un path analysis en base a las correlaciones y a las regresiones efectuadas previamente.

Para evaluar la bondad del ajuste del modelo a los datos se utilizaron los siguientes índices: el cociente entre $\chi^{2}$ y sus grados de libertad ( $\left.\chi^{2} / g l\right)$, el índice comparativo de Bentler-Bonett (CFI), el índice normalizado de Bentler-Bonett (NFI) y la raíz del error cuadrático medio de aproximación (RMSEA) de Steiger-Lind. Fueron considerados como aceptables los siguientes valores: $\chi^{2} / g l \leq 3$, CFI $\geq 0,95$, NFI $\geq 0,90$ y RMSEA $\leq 0,08$ (Ruiz, Pardo \& San Martín, 2010). Asimismo, en el presente estudio se asegura la pertinencia del análisis, debido a que la muestra es superior a los 200 casos y considerando que hay 10 variables introducidas en el modelo, lográndose satisfacer la recomendación de tener como mínimo 10 participantes por variable observada (Ruiz et al, 2010).

\section{Resultados}

\section{Análisis Descriptivo de las Escalas de Percepción del Sistema Normativo e Ideología Política}

En la Tabla 1 se pueden apreciar el promedio (y su intervalo de confianza) y desviación estándar de cada una de las dimensiones de las escalas de Percepción del Sistema Normativo y de ideología política.

Tabla 1

Media y Desviación Estándar de los Indicadores de Percepción del Sistema Normativo e Ideología Política

\begin{tabular}{lcccc}
\hline \multicolumn{1}{c}{ Variable } & $n$ & $M$ & $D E$ & $95 \%$ IC \\
\hline Percepción del sistema normativo & & & & \\
$\quad$ Falta de legitimidad & 155 & 5,59 & 0,62 & {$[5,48,5,70]$} \\
$\quad$ Transgresión & 196 & 5,84 & 0,84 & {$[5,72,5,92]$} \\
$\quad$ Debilidad normativa & 199 & 5,46 & 1,00 & {$[5,31,5,59]$} \\
Ideología política & & & & \\
$\quad$ Autoritarismo de derecha (RWA) & 189 & 3,42 & 0,80 & {$[3,30,3,53]$} \\
$\quad$ Orientación hacia la dominancia social (SDO) & 192 & 2,32 & 1,07 & {$[2,16,2,47]$} \\
\hline
\end{tabular}

Nota. El $n$ en cada dimensión es distinto, debido a que en algunas variables hay datos perdidos.

En la percepción del sistema normativo se aprecia una diferencia de los promedios de los tres factores, $F(2,302)=17,591, p<0,001, \eta_{\mathrm{p}}^{2}=0,104$, siendo el de la dimensión Transgresión más alto que el de Falta de Legitimidad $(p<0,001)$ y Debilidad Normativa $(p<0,001)$. En la variable ideología política se observa que el RWA obtuvo puntajes mayores que la SDO, $t(184)=13,557, p<0,001, d=1,168$, pero estos igual son relativamente bajos. Si se tiene en cuenta que la media teórica es de 4, se podrían considerar a las dimensiones del sistema normativo como por encima de ese valor y a las ideologías políticas autoritaria y dominante por debajo del mismo.

\section{Análisis Factorial y Descriptivo de la Escala de Cinismo Político}

Los datos presentaron una adecuación muestral aceptable para el análisis factorial exploratorio, $K M O=0,757, p<0,001$. En este se obtuvieron cinco dimensiones que explican el $65,85 \%$ de la varianza del cinismo político. En la Tabla 2 se muestran las cargas factoriales de los ítems a cada componente. 
Tabla 2

Ponderaciones de Factores para el Análisis Factorial Exploratorio de la Escala de Cinismo Político

\begin{tabular}{|c|c|c|c|c|c|}
\hline Ítem & 1 & 2 & 3 & 4 & 5 \\
\hline Ninguna persona que entra a la política puede seguir siendo honesta. & 0,816 & $-0,027$ & 0,143 & $-0,076$ & 0,062 \\
\hline Todos los políticos son malos, solo que algunos son peores que otros. & 0,774 & 0,022 & 0,229 & 0,049 & 0,048 \\
\hline $\begin{array}{l}\text { Es irrelevante por quién vote, ya que todos los candidatos son corruptos y esto } \\
\text { no va a cambiar. }\end{array}$ & 0,700 & 0,201 & $-0,058$ & 0,375 & $-0,127$ \\
\hline En nuestro país, si un político es eficiente, muy probablemente sea corrupto. & 0,643 & 0,308 & $-0,007$ & 0,115 & 0,017 \\
\hline Al sistema político en el Perú no lo cambia nadie. & 0,612 & 0,179 & $-0,208$ & 0,415 & $-0,185$ \\
\hline La política se rige sobre todo por el dinero. & 0,602 & $-0,036$ & 0,359 & 0,188 & $-0,138$ \\
\hline $\begin{array}{l}\text { Es legítimo elegir a aquellos candidatos que mejor respondan a mis intereses } \\
\text { personales, aunque sean corruptos. }\end{array}$ & 0,156 & 0,868 & $-0,073$ & $-0,061$ & $-0,094$ \\
\hline $\begin{array}{l}\text { Todos los políticos son malos, así que es mejor elegir aquellos que "roban pero } \\
\text { hacen obra". }\end{array}$ & 0,170 & 0,758 & 0,123 & $-0,030$ & $-0,263$ \\
\hline $\begin{array}{l}\text { Es válido elegir a aquellos candidatos que respondan a mis propios intereses, } \\
\text { aunque afecten los intereses de otros ciudadanos. }\end{array}$ & $-0,008$ & 0,735 & $-0,140$ & 0,024 & 0,102 \\
\hline La corrupción está muy ligada al sistema político en el país. & $-0,028$ & $-0,013$ & 0,875 & 0,163 & $-0,029$ \\
\hline Los políticos tienden a seguir sus propios intereses. & 0,307 & $-0,075$ & 0,748 & 0,010 & 0,152 \\
\hline Confío en que el sistema político actuará de manera eficiente. & 0,008 & 0,086 & $-0,247$ & $-0,721$ & $-0,122$ \\
\hline Siento que el sistema político peruano difícilmente mejorará. & 0,428 & 0,093 & $-0,129$ & 0,714 & $-0,098$ \\
\hline El sistema político en el país es malo. & 0,184 & $-0,117$ & 0,429 & 0,548 & 0,044 \\
\hline $\begin{array}{l}\text { Creo que todas las personas tenemos la capacidad de intervenir en la política } \\
\text { para limpiarla. }\end{array}$ & $-0,036$ & $-0,037$ & 0,074 & $-0,161$ & 0,817 \\
\hline $\begin{array}{l}\text { Ante un mal sistema político, es responsabilidad de los ciudadanos hacer cosas } \\
\text { para cambiarlo. }\end{array}$ & $-0,020$ & $-0,109$ & 0,012 & 0,221 & 0,781 \\
\hline \% de varianza explicada con rotación ortogonal & 20,452 & 13,043 & 11,752 & 11,283 & 9,324 \\
\hline
\end{tabular}

Nota. Las cargas factoriales resaltadas son aquellas mayores a 0,5 .

El primer componente obtenido se denominó Desconfianza en el Sistema Político $(a=0,83)$ y fue conformado por seis ítems referidos a una visión negativa hacia la política y a los actores políticos. Esta dimensión conlleva, asimismo, una idea generalizada de que el sistema no es confiable y que difícilmente puede ser cambiado. El segundo componente fue denominado Laxitud Moral Política $(\alpha=0,72)$ y agrupa tres ítems que hacen referencia a la intención de mantener un sistema político y actores políticos corruptos e ineficientes, en la medida en que estos respondan a los intereses de un individuo o grupo, sin considerar el daño o perjuicio que este ejercicio de la política produce en otros sectores de la sociedad. El tercer factor obtenido se denominó Corrupción en el Sistema Político $(a=0,72)$ y agrupa dos ítems asociados a una descripción más específica de la política y los políticos como corruptos. El cuarto factor fue denominado Ineficacia del Sistema Político $(\alpha=0,58)$ y está compuesto por tres ítems relacionados con una percepción de mal funcionamiento y malos resultados del sistema político peruano. Por último, el quinto componente fue denominado Posibilidad de Cambio del Sistema Político $(\alpha=0,54)$ y estuvo formado por dos ítems relacionados con la percepción de que es posible introducir cambios en un sistema político a través de la participación ciudadana. Los ítems con cargas factoriales negativas en sus respectivas dimensiones fueron invertidos para la construcción de los indicadores correspondientes. Los descriptivos de estas dimensiones se encuentran en la Tabla 3. 
Tabla 3

Media y Desviación Estándar de las Dimensiones del Cinismo Político

\begin{tabular}{lccc}
\hline \multicolumn{1}{c}{ Indicador } & $n$ & $M$ & $D E$ \\
\hline Desconfianza en el sistema político & 198 & 2,40 & 0,67 \\
Laxitud moral política & 198 & 1,48 & 0,62 \\
Corrupción en el sistema político & 197 & 3,60 & 0,57 \\
Ineficacia del sistema político & 196 & 3,11 & 0,60 \\
Posibilidad de cambio del sistema político & 197 & 3,43 & 0,64 \\
\hline
\end{tabular}

Nota. El $n$ en cada dimensión es distinto, debido a que en algunas variables hay datos perdidos.

\section{Relaciones entre la Percepción del Sistema Normativo, la Ideología Política y el Cinismo Político}

En la Tabla 4 se puede ver la relación entre los componentes de la percepción del sistema normativo, los de la ideología política y los del cinismo político, tomando en cuenta a todos los participantes del estudio.

Tabla 4

Relación entre la Percepción del Sistema Normativo, la Ideología Política y el Cinismo Político

\begin{tabular}{|c|c|c|c|c|c|c|c|c|c|}
\hline & 2 & 3 & 4 & 5 & 6 & 7 & 8 & 9 & 10 \\
\hline $\begin{array}{l}\text { 1. Falta de } \\
\text { legitimidad }\end{array}$ & $0,435^{* *}$ & $0,443^{* *}$ & 0,120 & $-0,194^{*}$ & 0,036 & $-0,333^{* *}$ & $0,373^{* *}$ & 0,057 & $0,313^{* *}$ \\
\hline 2. Transgresión & -- & $0,454^{* *}$ & 0,086 & 0,055 & $0,308^{* *}$ & $-0,116$ & $0,375^{* *}$ & $0,306^{* *}$ & 0,115 \\
\hline $\begin{array}{l}\text { 3. Debilidad } \\
\text { normativa }\end{array}$ & & -- & 0,117 & 0,002 & $0,254^{* *}$ & $-0,009$ & $0,160^{*}$ & $0,275^{* *}$ & $0,185^{* *}$ \\
\hline 4. RWA & & & -- & $0,344^{* *}$ & $0,313^{* *}$ & $0,234^{* *}$ & $-0,048$ & $-0,089$ & $-0,082$ \\
\hline 5. SDO & & & & -- & $0,182^{*}$ & $0,555^{* *}$ & $-0,101$ & $-0,052$ & $-0,217^{* * *}$ \\
\hline $\begin{array}{l}\text { 6. Desconfianza } \\
\text { sistema político }\end{array}$ & & & & & -- & $0,271^{* *}$ & $0,224^{* *}$ & $0,479^{* *}$ & $-0,099$ \\
\hline $\begin{array}{l}\text { 7. Laxitud moral } \\
\text { política }\end{array}$ & & & & & & -- & $-0,105$ & $-0,016$ & $-0,190^{* *}$ \\
\hline $\begin{array}{l}\text { 8. Corrupción } \\
\text { sistema político }\end{array}$ & & & & & & & -- & $0,301^{* *}$ & 0,111 \\
\hline $\begin{array}{l}\text { 9. Ineficacia sistema } \\
\text { político }\end{array}$ & & & & & & & & -- & 0,041 \\
\hline $\begin{array}{l}\text { 10. Cambio sistema } \\
\text { político }\end{array}$ & & & & & & & & & -- \\
\hline
\end{tabular}

En cuanto a las correlaciones entre los componentes de la Escala de Percepción del Sistema Normativo y las escalas de ideología política, solo se encontró una correlación inversa pequeña entre la dimensión Falta de Legitimidad y la SDO.

Por otro lado, las magnitudes del efecto, encontradas como resultado de las correlaciones entre las dimensiones de la Escala de Percepción del Sistema Normativo y las dimensiones de la Escala de Cinismo Político, tienen una intensidad pequeña y mediana, como se observa en la Tabla 4.

En cuanto a las relaciones entre la ideología política y el cinismo político, en la Tabla 4 se puede observar que existen cinco correlaciones pequeñas o medianas entre las variables.

Teniendo en cuenta que la media teórica es de 2,5, las dimensiones Corrupción en el Sistema Político, Necesidad de Cambio del Sistema Político e Ineficacia del Sistema Político son las que están por encima de este valor. Por su parte, tanto Desconfianza en el Sistema Político como Laxitud Moral Política, al ser las más bajas, se encuentran por debajo de dicha media. 


\section{Análisis de Regresión sobre los Efectos de la Ideología Política en la Percepción del Sistema Normativo}

Se desarrollaron tres análisis de regresión por pasos, considerando como variables predictoras las dimensiones ideológicas de RWA y SDO y como variables de criterio las dimensiones de la Escala de Percepción del Sistema Normativo. De los tres análisis realizados, se encontró solo un modelo significativo, que explica el 6,9\% de la varianza de la dimensión Falta de Legitimidad, $F(1,142)=5,275, p=0,006$, $R^{2}=0,069$. Se aprecia que la SDO, $b=-0,146, t=-2,895, p=0,004,95 \%$ IC $[-0,246,-0,046]$, y el RWA, $b=0,152, t=2,247, p=0,026,95 \%$ IC $[0,018,0,285]$ son predictores de dicha dimensión. Las dimensiones Transgresión y Debilidad Normativa no mostraron modelos significativos de regresión.

\section{Análisis de Regresión sobre los Efectos de la Ideología Política en el Cinismo Político}

De los cinco modelos elaborados, solo tres fueron significativos: (a) Desconfianza en el Sistema Político, (b) Laxitud Moral Política y (c) Necesidad de Cambio en el Sistema Político (como variables dependientes). El primero, en el que se excluye a la SDO como variable predictiva, explica un 9,7\% de la varianza, $F(1,182)=19,639, p<0,001, R^{2}=0,097$, de manera que se puede apoyar la hipótesis de que el RWA ejerce influencia directa en la desconfianza en el sistema político, $b=0,258, t=4,432, p<0,001,95 \%$ IC $[0,143,0,372]$. El segundo modelo, en el que se excluye al RWA como variable predictiva, explica un 30,2\% de la varianza, $F(1,182)=79,096, p<0,001, R^{2}=0,302$, por lo que se puede apoyar la idea de que la SDO influye en la laxitud moral, $b=0,303, t=8,894, p<0,001,95 \%$ IC [0,236, 0,370]. Finalmente, el tercer modelo, en el que se excluye al RWA como variable predictiva, explica un 4,7\% de la varianza, $F(1,181)=8,836, p=0,003$, $R^{2}=0,047$, de manera que la SDO resulta ser el mejor predictor de la necesidad de cambio en el sistema político, $b=-0,124, t=-2,973, p=0,003,95 \%$ IC [-0,207, -0,042].

\section{Análisis de Regresión sobre los Efectos de la Percepción del Sistema Normativo en el Cinismo Político}

Se proponen cinco modelos, uno para cada dimensión del cinismo político. El primer modelo, en el que se excluyó la falta de legitimidad y la debilidad normativa como variables predictivas, explica el 7,3\% de la varianza de la desconfianza en el sistema político, $F(1,149)=11,739, p=0,001, R^{2}=0,073$. Se encontró que la transgresión es la única que influye directamente en dicha variable, $b=0,220, t=3,426, p=0,001$, $95 \%$ IC $[0,093,0,347]$.

El segundo modelo, en el que se excluyó la falta de legitimidad y la transgresión como variables predictoras, explica el 9,9\% de la varianza de la laxitud moral política, $F(1,149)=16,423, p<0,001$, $R^{2}=0,099$, quedando únicamente la debilidad normativa como el mejor predictor, $b=-0,286, t=-4,053$, $p<0,001,95 \%$ IC [-0,426, - 0,147].

A su vez, el tercer modelo, en el que se excluyó a la dimensión Debilidad Normativa, explica el 17,2\% de la varianza de la corrupción en el sistema político, $F(1,147)=15,244, p<0,001, R^{2}=0,172$, siendo la falta de legitimidad, $B=0,231, t=3,379, p=0,001,95 \%$ IC [0,096, 0,366], y la transgresión, $b=0,143, t=2,409$, $p=0,017,95 \%$ IC $[0,026,0,261]$, los factores que influyen en la variable dependiente en cuestión.

Por su parte, el cuarto modelo, en el que se excluyó la falta de legitimidad y la transgresión como variables predictivas, explica el 4,1\% de la varianza de la dimensión Ineficacia en el Sistema Político, $F(1,147)=6,222$, $p=0,014, R^{2}=0,041$. La debilidad normativa sería el mejor predictor de la variable antes mencionada, $b=0,127, t=2,494, p=0,014,95 \%$ IC [0,026, 0,228].

Por último, el quinto modelo propuesto, excluyéndose las dimensiones Transgresión y Debilidad Normativa, explica el 9,3\% de la varianza del cambio en el sistema político, $F(1,149)=15,256, p<0,001$, $R^{2}=0,093$. Se encontró que la falta de legitimidad es el único factor que ejerce influencia en dicha dimensión del cinismo político, $b=0,281, t=3,906, p<0,001,95 \%$ IC $[0,139,0,423]$.

\section{Path Analysis: Cinismo Político como Variable Dependiente}

Por medio del path analysis realizado, se propone un modelo en base a lo previamente observado (ver Figura 1), el cual muestra un buen ajuste según los siguientes índices: $\chi^{2} / g l=1,703, \mathrm{CFI}=0,953, \mathrm{NFI}=0,901$, RMSEA $=0,059,90 \%$ IC $[0,000,0,996]$. 


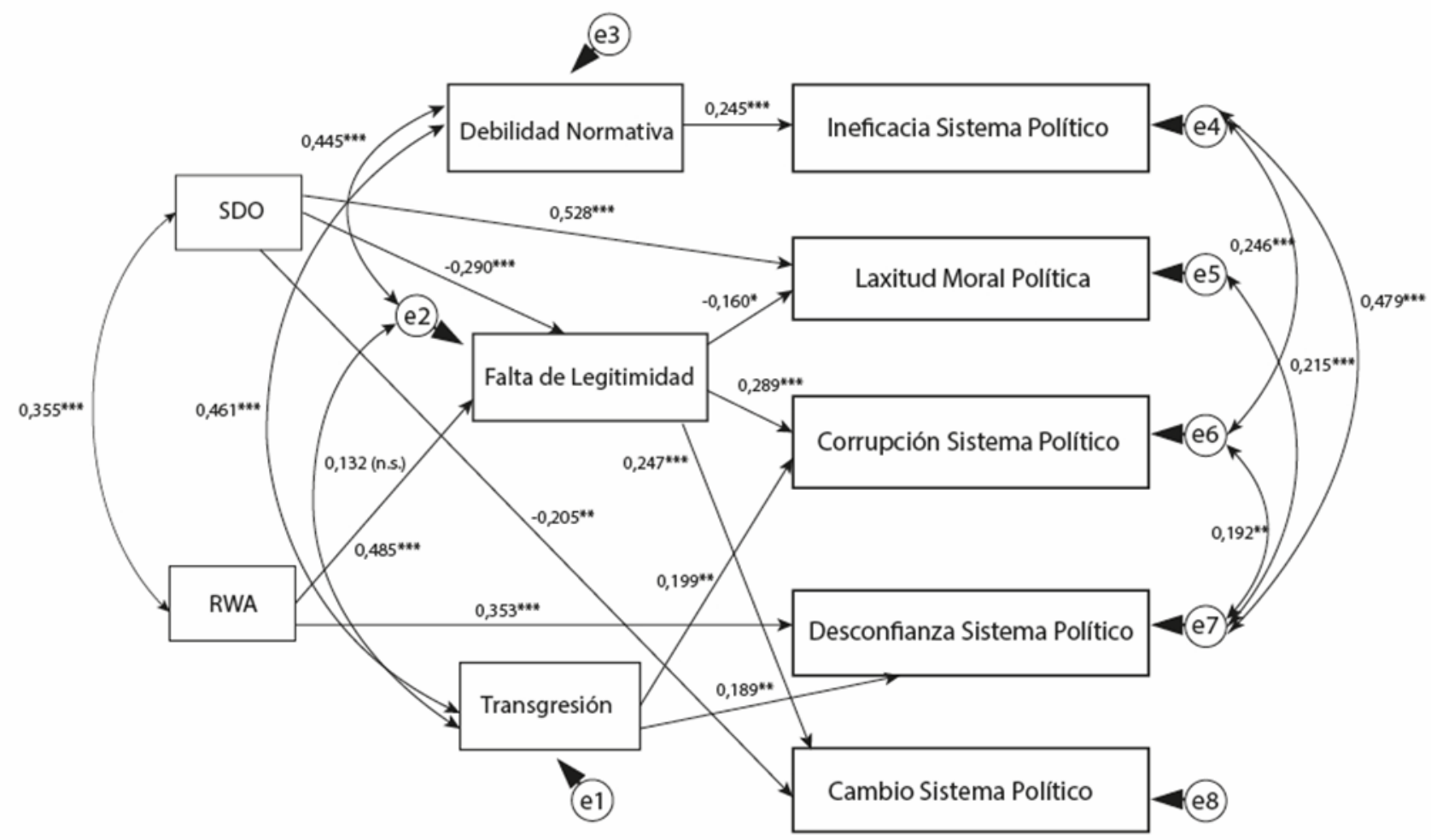

Figura 1. Diagrama del path analysis del modelo propuesto. ${ }^{*} p<0,05 ;{ }^{*} p<0,01 ; * * * p<0,001$; (n.s.) = marginalmente significativo.

Es pertinente señalar que, después de la revisión teórica de la relación entre las variables, en el modelo trabajado se utiliza como variable dependiente el cinismo político, debido a la repercusión que tiene la ideología política y la percepción del sistema normativo en la variable en cuestión.

El modelo propuesto presenta una lógica deductiva basada en la literatura, donde los indicadores ideológicos, por su nivel de abstracción, aparecen en un primer nivel de análisis como variables exógenas, las cuales influencian un segundo nivel de análisis, en el que se encuentran las dimensiones correspondientes a las percepciones generales sobre el sistema normativo e institucional del país. Las dimensiones ideológicas y las percepciones sobre el sistema normativo inciden, a su vez, en un tercer nivel analítico, conformado por las percepciones y actitudes sobre el sistema político peruano, entendido en el presente estudio como un objeto de representación más específico.

\section{Discusión}

En primer lugar, los resultados dan cuenta de una visión predominantemente negativa del sistema normativo. De manera específica, las puntuaciones en las dimensiones de la escala sugieren una alta percepción de transgresión de las normas en la sociedad y falta de legitimidad percibida en las instituciones encargadas de promover el orden, lo cual genera una visión de un sistema normativo débil y frágil. Los sistemas normativos tienden a ser percibidos negativamente en sociedades donde la corrupción y la transgresión se han constituido en prácticas recurrentes a lo largo de su historia (Beramendi, 2014; Beramendi \& Zubieta, 2013). De tal manera, parece ser que un sistema normativo como el peruano, con una historia larga de corrupción y vulneración de normas y leyes, produce en sectores importantes de su ciudadanía una tendencia a naturalizar la presencia de estas prácticas como algo normal o inevitable (Quiroz, 2008/2013).

$\mathrm{Al}$ mismo tiempo, estas prácticas tienen efectos en aspectos tales como la confianza institucional e interpersonal en una sociedad, así como mayores niveles de anomia o falta de interés en asuntos sobre los 
que se tomen decisiones políticas que, se supone, podrían ayudar a cambiar estos fenómenos que debilitan la convivencia en una sociedad (Beramendi \& Zubieta, 2013; Muratori, Delfino \& Zubieta, 2013; Strama, 1998). Sobre este aspecto, adquiere relevancia la idea del cinismo político; el estudio del cinismo político está caracterizado por los efectos de este fenómeno en los niveles de confianza hacia un sistema (Miller, 1974; Siukai, 1992). Sin embargo, un aporte significativo de la presente investigación es que complementa la visión original del concepto con dimensiones relevantes para el análisis del mismo. Así, el análisis de estructura de la Escala de Cinismo Político desarrollada para el presente estudio corrobora la presencia de una dimensión de desconfianza general hacia el sistema, así como percepciones de ineficacia y corrupción en el mismo.

Ahora, sobre estas dimensiones de la escala, los resultados sugieren dos nuevas dimensiones que pueden interpretarse como consecuencias del cinismo, más allá de la mera desconfianza hacia el sistema político: (a) la idea de que las deficiencias del sistema político pueden ser aprovechadas para beneficio personal, en una dimensión que se ha denominado Laxitud Moral Política (aunque en la muestra del presente estudio esta dimensión se encuentra bajo el promedio teórico) y (b) la idea de que el sistema político es efectivamente deficiente y que es posible tomar acciones desde la ciudadanía para cambiarlo, lo cual iría en contra de la idea de que el cinismo normalmente se caracteriza por el escepticismo ante una posibilidad de cambio (Fu et al., 2011). En ese sentido, se puede apreciar una especie de dualidad del cinismo político. En primer lugar, existe una concepción negativa que va más allá de un desinterés en la participación política y que implicaría una visión acrítica del sistema y, al mismo tiempo, fomentaría una relación clientelar con el mismo. Por otro lado, coexiste esta visión positiva en donde los ciudadanos tienen que buscar de manera activa una transformación de la sociedad y así evitar que sucedan eventos considerados como perjudiciales para la salud de la sociedad.

En la actualidad política del Perú, las dos dimensiones reportadas encuentran sustento en ejemplos de la vida política cotidiana. En las elecciones generales del 2006 y el 2011, Ollanta Humala, que representaba una candidatura para una gran transformación del país, recibió la mayoría de sus votos de sectores de la población menos favorecidos o que representaban políticamente la posición por un cambio (Espinosa, 2008), mientras que en el 2006, Alan García y en el 2011, Keiko Fujimori (las opciones asociadas al mantenimiento del sistema) recibieron un voto más urbano y de sectores socialmente más favorecidos (Tanaka, Barrenechea \& Vera, 2011), motivados por el miedo al cambio. En ambos casos, los discursos de los electores de García y Fujimori daban cuenta de la persistencia de una organización jerárquica que permite que aquellas personas que se consideran más poderosas validen, a través de su discurso y voto, la centralización del poder político y económico, encontrándose, entonces, a favor del mantenimiento de un modelo económico y social que ofrecería una relación clientelar con el eventual futuro gobierno (Ferrándiz, Ibáñez \& Espinosa, 2011; Guevara Rojas \& Espinosa, 2014).

De la misma forma, el escenario electoral del 2016 representa un claro ejemplo de la dinámica mencionada. La última candidatura de Keiko Fujimori despertó un enfrentamiento en la opinión pública que puso en evidencia ambas posiciones. Por un lado, la alta aceptación de la candidata —quien alcanzó el 49,41\% de los votos en la segunda vuelta electoral (Elecciones Perú, 2016, Junio 6) - permite reflexionar sobre la alta aceptación entre los votantes peruanos de una apuesta política autoritaria y con un pasado de violencia y corrupción del que no se deslinda. Sin embargo, como contrapartida, se da el surgimiento de colectivos antifujimoristas, así como la acogida de movimientos como las marchas "Keiko no va", los cuales surgen en el 2009 y cuya última marcha en mayo del 2016 concentró 70 mil manifestantes (Mendoza, 2016, Mayo 31), lo que daría cuenta de la existencia de una posición más liberal y cuestionadora de los riesgos que supone persistir en la elección de candidaturas vinculadas a un pasado reciente de violencia y corrupción.

De manera conceptual, ello refleja dos elementos centrales que diferencian el liberalismo del conservadurismo: el apoyo (versus el rechazo) al cambio social y el rechazo (versus el soporte) a la inequidad social (cf. Jost et al., 2003; Jost et al., 2009), así como también ejemplifica con claridad la coexistencia en la sociedad peruana de una visión crítica y una resistencia al cambio (a favor de la preservación de ventajas personales). Esta reflexión coincide con los hallazgos de Schmitz y Espinosa (2015), quienes, dentro de un contexto polarizado, detectaron un sector menos conservador entre los limeños, el cual estaba a favor de los cambios en la sociedad y la disminución de las jerarquías sociales.

En base a lo anteriormente mencionado, queda responder a la siguiente pregunta: ¿cómo se asocia la ideología a la percepción de los sistemas normativos y a la presencia del cinismo político? En base al modelo propuesto, se aprecia que, de los indicadores ideológicos, solo la SDO estaría influyendo en una dimensión de la Escala de Percepción del Sistema Normativo. Específicamente, los resultados sugieren que, a mayor 
dominancia, menor sería la percepción de falta de legitimidad en el sistema, lo que significaría que, a pesar de que existe acuerdo con una percepción mayoritariamente negativa del sistema normativo, los dominantes tienden a cuestionar menos la legitimidad del mismo (Jackson \& Gaertner, 2010). Esto es consistente con la idea de que la legitimidad percibida en el sistema se construye, en el caso de los dominantes, desde una perspectiva acrítica y utilitaria del tipo "si el sistema tal como es, me es útil, está bien". Así, aquellas personas más dominantes enfocan su percepción de la eficacia del sistema normativo en relación con cuánto este aporta al mantenimiento de su posición y ventajas sociales (Rottenbacher \& Schmitz, 2012). Dicha información se corrobora, a nivel teórico, con estudios que relacionan la dominancia social no solo con la percepción del sistema normativo, sino también con la intención transgresora, encontrándose que individuos dominantes tienden a ser más propensos a presentar y justificar actos de transgresión y corrupción (Gnädinger, 2014; Rottenbacher \& Schmitz, 2012).

Al mismo tiempo, este resultado también es coherente con la relación directa obtenida entre la dimensión del cinismo político asociada a la laxitud moral y la SDO, de tal forma que aquellas personas más dominantes estarían dejando de lado una perspectiva moral a cambio del cumplimiento de sus deseos (Jackson \& Gaertner, 2010). Este resultado reafirma lo planteado por Shannon (2000), quien menciona que, al encontrarse ante un dilema moral entre sus deseos y las restricciones sociales, las personas interpretan las normas y situaciones de tal manera que puedan actuar según sus deseos, sin sentirse afectados en el plano individual.

Es también posible vislumbrar estos resultados a partir de dos aristas diferentes. Por un lado, el deseo de poder, predominante en aquellas personas más dominantes, motivaría la transgresión de un sistema poco validado; mientras que, por otro lado, la transgresión de aquellos más dominantes también podría partir de la sensación de omnipotencia que su actual posición de poder les otorga (Rottenbacher \& Schmitz, 2012), debido a que consideran que no tendrán sanción alguna.

Por otra parte, el autoritarismo de derecha resulta el mejor predictor de la dimensión del cinismo político que se refiere a la desconfianza en el sistema político. Esta relación guarda sentido, dado que las personas más autoritarias tienden a ser más susceptibles a la amenaza y, por tanto, a la desconfianza (Jackson \& Gaertner, 2010). La historia de transgresión y corrupción ligada al Estado peruano daría fundamento suficiente para la desconfianza, más aún para aquellas personas más susceptibles a la misma (Quiroz, 2008/2013). A pesar de ello, el mal funcionamiento del sistema normativo y político estaría tan arraigado en la tradición autoritaria del país que las personas más autoritarias no se adherirían a la necesidad de cambiarlo.

En la historia del Perú, periodos de crisis e inestabilidad se vivieron como antecesores a gobiernos autoritarios (e.g., Oncenio de Leguía y el fujimorato) (Flores Galindo, 1999), lo cual habría generado terreno fértil entre la población más conservadora para la aceptación de este tipo de gobiernos a cambio de una idea de control y estabilidad, aceptando incluso altos niveles de corrupción. Esta situación, sumada a la tradición autoritaria en el país, habría sentado las bases para que este tipo de sistemas sea percibido por los más autoritarios - tal como lo indican los resultados del presente estudio y los últimos episodios de la historia política del país - como un sistema válido para la conservación de la seguridad y estabilidad.

Los resultados del presente estudio muestran la complejidad del escenario político y social peruano, así como una situación ambivalente frente a la percepción del sistema normativo y la posibilidad de un accionar hacia el cambio, que implicaría tanto factores ideológicos como históricos y contextuales. Si bien la percepción negativa observada supone una aproximación crítica por parte de los participantes al funcionamiento del sistema político y las instituciones en el país, esta perspectiva y la desconfianza en el sistema político no serían suficientes para motivar una necesidad de cambio lo suficientemente sólida.

Por el contrario, los niveles de cinismo político, más allá de la desconfianza en el sistema que implica, darían cuenta de la posibilidad de una resistencia al cambio en el sistema. Esto se genera debido a una visión egoísta y orientada hacia el propio beneficio de ciertos sectores, así como la conservación de un sistema ya establecido en la tradición autoritaria. Así, ciertas manifestaciones tanto autoritarias como dominantes tienden a agravar la situación de cinismo político y la percepción negativa del sistema normativo, consolidándose un círculo vicioso que incluso podría motivar un debate moral cuando se encuentran ante la situación de elegir un modelo económico que les resulta conveniente, a pesar de la tradición de corrupción de los sectores más conservadores que ciertas candidaturas representan.

En cuanto a las limitaciones del estudio, se considera que las variables sociodemográficas de nivel socioeconómico, nivel de estudios, edad y sexo, que solo se tuvieron en cuenta en este estudio para describir 
a la muestra, deberían utilizarse como variables de segmentación. En este sentido, se plantea que en futuros estudios se consideren dichas variables para analizar su posible efecto o condicionamiento en la percepción del sistema político y social del país. Al mismo tiempo, al tratarse de una muestra accidental, los resultados no pueden ser generalizados. Adicionalmente, se reconoce como una limitación del estudio el hecho de que dos factores del cinismo político obtuvieron una baja consistencia interna, por lo que se recomienda la realización de futuros estudios que incorporen más ítems que permitan mejorar dicha consistencia.

\section{Referencias}

Altemeyer, B. (1998). The other “autoritarian personality". Advances in Experimental Social Psychology, 30, 47-92. https://doi.org/10.1016/S00652601(08)60382-2

Bélanger, E. \& Aarts, K. (2006). Explaining the rise of the LPF: Issues, discontent and the 2002 Dutch election. Acta Politica, 41, 4-20. https://doi.org/10.1057/palgrave.ap.5500135

Beramendi, M. (2014). Percepción del sistema normativo, transgresión y sus correlatos psicosociales en Argentina (Tesis de Doctorado no publicada), Facultad de Psicología, Universidad de Buenos Aires, Argentina.

Beramendi, M. \& Zubieta, E. (2013). Norma perversa: transgresión como modelado de legitimidad. Universitas Psychologica, 12, 591600. https://doi.org/10.11144/Javeriana.UPSY12-2.nptm

Beramendi, M. \& Zubieta, E. (2014). Construcción y validación de la Escala de Percepción del Sistema Normativo. Revista Mexicana de Psicología, 31, 124-137. Extraído de http://www.redalyc.org/pdf/2430/243033031005.pdf

Brown, R. (2004). The authoritarian personality and the organization of attitudes. En J. T. Jost \& J. Sidanius (Eds.), Political Psychology: Key readings (pp. 39-68). New York, NY: Psychology Press.

Cohen, J. (1988). Statistical power analysis for the behavioral sciences (2a ed.). Mahwah, NJ: Lawrence Erlbaum.

Duckitt, J. (2001). A dual-process cognitive-motivational theory of ideology and prejudice. Advances in Experimental Social Psychology, 33, 41-113. https://doi.org/10.1016/S0065-2601(01)80004-6

Duckitt, J. \& Fisher, K. (2003). The impact of social threat on worldview and ideological attitudes. Political Psychology, 24, 199-222. https://doi.org/10.1111/0162-895X.00322

Duckitt, J. \& Sibley, C. G. (2007). Right wing authoritarianism, social dominance orientation and the dimensions of generalized prejudice. European Journal of Personality, 21, 113-130. https://doi.org/10.1002/per.614

Duckitt, J, Wagner, C, du Plessis, I. \& Birum, I. (2002). The psychological bases of ideology and prejudice: Testing a dual process model. Journal of Personality and Social Psychology, 83, 75-93. https://doi.org/10.1037/0022-3514.83.1.75

Duriez, B, Van Hiel, A. \& Kossowska, M. (2005). Authoritarianism and social dominance in Western and Eastern Europe: The importance of the sociopolitical context and of political interest and involvement. Political Psychology, 26, 299-320. https://doi.org/10.1111/j.1467-9221.2005.00419.x

Elecciones Perú 2016: revive segunda vuelta entre PPK y Keiko (2016, Junio 6). El Comercio, Política. Extraído de http://elcomercio.pe/politica/elecciones/elecciones-2016-vivo-keiko-fujimori-y-ppk-disputan-segunda-vuelta-noticia-1906794

Espinosa, A. (2008). Decidiéndose por el mal menor: el rol de las emociones en las elecciones peruanas del 2006. Psicología Política (Valencia,España), 37, 47-70. Extraído de http://www.uv.es/garzon/psicologia\%20politica/N37-3.pdf

Espinosa, A. (2011). Estudios sobre identidad nacional en el Perú y sus correlatos psicológicos, sociales y culturales (Tesis de doctorado no publicada), Departamento de Psicología Social y Metodología de las Ciencias del Comportamieno, Universidad del País VascoEuskal Herriko Unibertsitatea, Donostia/San Sebastián, España.

Ferrándiz, J., Ibáñez, C. \& Espinosa, A. (2011). Racismo 2.0: expresiones de prejuicio en las redes sociales virtuales tras las elecciones generales de 2011. Politai, 2(3), 75-83. Extraído de http://revistas.pucp.edu.pe/index.php/politai/article/view/13954/14577

Flores Galindo, A. (1999) La tradición autoritaria: violencia y democracia en el Perú. Lima, Perú: Asociación Pro Derechos Humanos/SUR

Fu, H., Mou, Y., Miller, M. J. \& Jalette, G. (2011) Reconsidering political cynicism and political involvement: A test of antecedents. American Communication Journal, 13(2), 44-61. Extraído de http://ac-journal.org/journal/pubs/2011/summer/Cynicism_Proof.pdf

Gächter, S. \& Schulz, J. F. (2016). Intrinsic honesty and the prevalence of rule violations across societies. Nature, 531, $496-499$. https://doi.org/10.1038/nature17160

Gnädinger, M. (2014). Identidad nacional, tolerancia a la trangresión e ideologías políticas en adultos residentes en Lima (Tesis de grado no publicada), Facultad de Letras y Ciencias Humanas, Pontificia Universidad Católica del Perú, Lima, Perú.

Guevara Rojas, L. A. \& Espinosa, A. (2014). Estereotipos y emociones intergrupales en Facebook durante las elecciones generales peruanas del 2011. Revista Electrónica de Psicología Política, 33, 25-48. Extraído de http://www.psicopol.unsl.edu.ar/b2014Diciembre-Art\%EDculo2.pdf

Jackson, L. E. \& Gaertner, L. (2010). Mechanisms of moral disengagement and their differential use by right-wing authoritarianism and social dominance orientation in support of war. Aggressive Behavior, 36, 238-250. https://doi.org/10.1002/ab.20344

Jost, J. T. (2006). The end of the end of ideology. American Psychologist, 61, 651-670. https://doi.org/10.1037/0003-066X.61.7.651

Jost, J. T., Federico, C. M. \& Napier, J. L. (2009). Political ideology: Its structure, functions, and elective affinities. Annual Review of Psychology, 60, 307-337. https://doi.org/10.1146/annurev.psych.60.110707.163600

Jost, J. T., Glaser, J., Kruglanski, A. W. \& Sulloway, F. J. (2003). Political conservatism as motivated social cognition. Psychological Bulletin, 129, 339-375. https://doi.org/10.1037/0033-2909.129.3.339

Kelman, H. C. (2001). The role of national identity in conflict resolution: Experiences from Israeli-Palestinian problem-solving workshops. En R. D. Ashmore, L. Jussim \& D. Wilder (Eds.), Social identity, intergroup conflict, and conflict reduction (pp. 187212). New York, NY: Oxford University Press.

Knight, K. (2006). Transformations of the concept of ideology in the twentieth century. American Political Science Review, 100, 619-626. https://doi.org/10.1017/S0003055406062502

Latinobarómetro (2015). Apoyo a la democracia: Perú. Santiago, Chile: Autor. Extraído de http://www.latinobarometro.org/latOnline.jsp 
Major, B. \& Schmader, T. (2001). Legitimacy and the construal of social disadvantage. En J. T. Jost \& B. Major (Eds.), The psychology of legitimacy: Emerging perspectives on ideology, justice, and intergroup relations (pp. 176-204). New York, NY: Cambridge University Press

Mendoza, V. (2016, Mayo 31). Marcha 'Keiko no va': así fue la movilización contra la candidatura de Keiko Fujimori. La República, Política. Extraído de http://larepublica.pe/politica/772365-marcha-no-keiko-miles-se-manifiestan-hoy-en-el-peru-y-el-mundo

Miller, A. H. (1974). Political issues and trust in government: 1964-1970. American Political Science Review, 68, 951-972. https://doi.org/10.2307/1959140

Monsegur, S., Espinosa, A. \& Beramendi, M. (2014) Identidad nacional y su relación con la dominancia social y la tolerancia a la trangresión en residentes de Buenos Aires (Argentina). Interdisciplinaria, 31, 5-23. https://doi.org/10.16888/interd.2014.31.1.1

Moya, M. \& Morales-Marente, E. (2005). Reacciones psico-políticas ante los ataques terroristas del 11 de Marzo de 2004 . Revista de Psicología Social, 20, 331-350. https://doi.org/10.1174/021347405774277749

Muratori, M., Delfino, G. I. \& Zubieta, E. M. (2013). Percepción de anomia, confianza y bienestar: la mirada desde la psicología social. Revista de Psicología Pontificia Universidad Católica del Perú, 31, 129-150. Extraído de http://www.redalyc.org/pdf/3378/337829524005.pdf

Patterson, T. E. (2002) The vanishing voter: Public involvement in an age of uncertainty. New York, NY: Alfred A. Knopf.

Porter, J. (2007). Using structural equation modeling to examine the relationship between political cynicism and right-wing authoritarianism. Sociological Spectrum, 28, 36-54. https://doi.org/10.1080/02732170701675128

Pratto, F., Sidanius, J., Stallworth, L. M. \& Malle, B. F. (1994). Social dominance orientation: A personality variable predicting social and political attitudes. Journal of Personality and Social Psychology, 67, 741-763. https://doi.org/10.1037/0022-3514.67.4.741

Quiroz, A. W. (2008/2013). Historia de la corrupción en el Perú (J. Flores Espinoza, Trad.; Título original: Corrupt circles: A history of unbound graft in Peru). Lima, Perú: Instituto de Estudios Peruanos. https://doi.org/10.15446/achsc.v41n2.48794

Rottenbacher, J. M. \& Schmitz, M. (2012). Conservadurismo político y tolerancia hacia comportamientos transgresores. Psicología Política, 44, 31-56. Extraído de http://www.uv.es/garzon/psicologia\%20politica/N44-2.pdf

Ruiz, M. A., Pardo, A. \& San Martín, R. (2010). Modelos de ecuaciones estructurales. Papeles del Psicólogo, 31, 34-45. Extraído de http://www.redalyc.org/articulo.oa?id=77812441004

Schmitz, M. \& Espinosa, A. (2015). Ideología, emocionalidad y crisis de gobernabilidad en Lima Metropolitana: un estudio correlacional sobre el proceso de revocatoria del 2013. Revista Electrónica de Psicología Política, 34, 46-63. Extraído de http://www.psicopol.unsl.edu.ar/2015Julio-Art\%EDculo03.pdf

Shannon, V. P. (2000). Norms are what states make of them: The political psychology of norm violation. International Studies Quarterly, 44, 293-316. https://doi.org/10.1111/0020-8833.00159

Sidanius, J., Pratto, F. \& Bobo, L. (1996). Racism, conservatism, affirmative action, and intellectual sophistication: A matter of principled conservatism or group dominance? Journal of Personality and Social Psychology, 70, 476-490. https://doi.org/10.1037/00223514.70.3.476

Sidanius, J., Pratto, F., van Laar, C. \& Levin, S. (2004). Social dominance theory: Its agenda and method. Political Psychology, 25, 845880. https://doi.org/10.1111/j.1467-9221.2004.00401.x

Siu-kai, L. (1992). Decline of governmental authority, political cynicism and political inefficacy in Hong Kong. Journal of Northeast Asian Studies, 11(2), 3-20. https://doi.org/10.1007/BF03023340

Stone, W. F. (2001). Manipulación del terror y autoritarismo. Psicología Política, $23, \quad 7-17 . \quad$ Extraído de http://www.uv.es/garzon/psicologia\%20politica/N23-1.pdf

Strama, M. (1998). Overcoming cynicism: Youth participation and electoral politics. National Civic Review, 87, 71-78. https://doi.org/10.1002/ncr.87106

Tanaka, M., Barrenechea, R. \& Vera, S. (2011). Cambios y continuidades en las elecciones presidenciales de 2011. Argumentos: Revista de Análisis y Crítica (IEP), 5(2), 1-8. Extraído de http://revistaargumentos.iep.org.pe/articulos/cambios-y-continuidades-en-laselecciones-presidenciales-2011/

Tyler, T. R. (2001). Public trust and confidence in legal authorities: What do majority and minority group members want from the law and legal institutions? Behavioral Sciences \& the Law, 19, 215-235. https://doi.org/10.1002/bsl.438

Tyler, T. R. (2006). Psychological perspectives on legitimacy and legitimation. Annual Review of Psychology, 57, 375-400. https://doi.org/10.1146/annurev.psych.57.102904.190038

Van Hiel, A. \& Mervielde, I. (2002). Explaining conservative beliefs and political preferences: A comparison of social dominance orientation and authoritarianism. Journal of Applied Social Psychology, 32, 965-976. https://doi.org/10.1111/j.15591816.2002.tb00250.x

Zakrisson, I. (2005). Construction of a short version of the Right-Wing Authoritarianism (RWA) scale. Personality and Individual Differences, 39, 863-872. https:// doi.org/10.1016/j.paid.2005.02.026

Zelditch Jr., M. (2001). Theories of legitimacy. En J. T. Jost \& B. Major (Eds.), The psychology of legitimacy: Emerging perspectives on ideology, justice, and intergroup relations (pp. 33-53). New York, NY: Cambridge University Press.

Fecha de recepción: Marzo de 2017.

Fecha de aceptación: Octubre de 2017. 